$\begin{array}{cc}\text { Programa de Pós-Graduação em Engenharia de Produção - PPGEP } & \\ \text { Laboratório de Qualidade de Vida - LaQVida } & \text { REVISTA BRASILEIRA DE } \\ \text { Universidade Tecnológica Federal do Paraná - UTFPR } & \text { Ponta Grossa - PR - Brasil } \\ \text { v. 02, n. 02, jul./dez. 2010, p. } 01-08 & \text { QUALIDADE DE VIDA }\end{array}$

DOI: $10.3895 / \mathrm{S} 2175-08582010000200001$

\title{
Período de estudos e qualidade de vida do estudante de enfermagem
}

\section{Study period and quality of life of nursing students}

\author{
Natã Amaral de Sousa \\ Curso de Enfermagem da Universidade de Santo Amaro - UNISA - São Paulo - SP - Brasil \\ naatoon@ @hotmail.com \\ Isaac Rosa Marques \\ Curso de Enfermagem da Universidade de Santo Amaro - UNISA - São Paulo - SP - Brasil \\ imarques@unisa.br
}

\begin{abstract}
Resumo
O estudo teve como objetivo investigar a qualidade de vida (QV) de estudantes de enfermagem de uma universidade privada da cidade de São Paulo. Tratou-se de um estudo correlacional cuja variável de comparação foi o período de estudos. A amostra foi constituída por 230 estudantes de enfermagem, utilizando o instrumento WHOQOL-bref. Resultados demonstraram predominância de sujeitos do sexo feminino (81,7\%), da faixa etária de 21 a 25 anos $(41,3 \%)$. A QV referente aos cinco domínios do instrumento apresentou QV de nível intermediário mais frequente com os estudantes do período matutino $(52,5 \%)$ e QV de nível ótima com os estudantes do período noturno $(48,4 \%)$. Concluiu-se que não houve diferença estatisticamente significante na QV dos estudantes de enfermagem considerando o período de estudos.
\end{abstract}

Palavras-chave: qualidade de vida, estudante de enfermagem, graduação em enfermagem.

\begin{abstract}
The purpose of this study was to investigate the quality of life (QoL) of nursing students from a private university in São Paulo, SP, Brazil. A correlation study was carried out whose comparing variable was the period of study. The sample was constituted by 230 nursing students using the WHOQOL-bref. Results demonstrated a predominance of female individuals $(81,7 \%)$, whose range age was from 21 to 25 years-old $(41,3 \%)$. The QoL in the five domains showed QoL intermediate level more frequent for students of the morning period $(52,5,4 \%)$, and QoL high level more frequent for students of the night period $(48,4 \%)$. It was concluded that there are no significant differences in QoL considering the study period.
\end{abstract}

Keywords: quality of life, nursing students, nursing undergraduate.

\section{Introdução}

A Qualidade de Vida (QV) é considerada como boa ou excelente é aquela que oferece um mínimo de condições para que os indivíduos nela inseridos possam desenvolver o máximo de suas potencialidades, sejam estas: viver, sentir, amar, trabalhar, produzindo bens e serviços, fazendo ciência ou artes (NETTO, 1994).

A QV é composta de, pelo menos, seis domínios: o físico, o psicológico, o nível de independência, as relações sociais, o meio ambiente e a espiritualidade. A QV também abrange 
muitos significados que refletem conhecimentos, experiências e valores de indivíduos e coletividades que a ele se reportam em variadas épocas, espaços e histórias diferentes, sendo, portanto uma construção social com a marca da relatividade cultural indispensável a todo ser humano e essencial em tudo o que nos propusermos a realizar (THE WHOQOL GROUP, 1995).

Estudar a QV no ambiente universitário é de suma importância, pois engloba aspectos fundamentais no processo de aprendizagem, despertando o interesse no aluno, promovendo motivação para adquirir novos conhecimentos e interações com o a sua formação. Além disto, o discurso sobre o lazer e qualidade de vida vem crescendo de forma expressiva em nossa sociedade. A valorização destas esferas nos dias atuais pode ser percebida de forma geral em todas as camadas sociais e é parte integrante e fundamental no desenvolvimento chamado de globalização (JOLY; SANTOS; SISTO, 2005).

Ao considerar esta importância, para o graduando de enfermagem é também importante que exista um grande acolhimento por parte da instituição, sendo este espaço promotor ou não de vivências que contribuem para qualidade de vida dos estudantes e até mesmo sua formação profissional. Para o estudante, em geral, o ingresso no meio universitário o coloca diante de situações de crises acidentais, uma vez que sai do seu ambiente familiar e se depara com um mundo desconhecido podendo viver vários conflitos (CAPLAN, 1980; FIGUEIREDO; OLIVEIRA, 1995). Vale ressaltar que o Comitê de Ensino Superior de Enfermagem do Estado de São Paulo (CESE-SP) valoriza a contextualização do aluno e de suas condições de vida, classificando os como aspectos importantes no desenvolvimento de propostas para melhoria do ensino da profissão de enfermagem (TAVARES et al., 1995).

Em revisão da literatura, constatou-se que as escolas têm se baseado na assimilação ou não, de conteúdos informativos e menos no processo formativo (CIAMPONE, 1998). Nesse contexto, o papel do docente tem sido destacado como de grande importância quando este atua como facilitador para a adaptação do aluno aos primeiros contatos com o aprendizado prático da enfermagem (ÂNGELO, 1989). O crescimento no número de cursos de enfermagem trouxe também a possibilidade de realização do curso em períodos vespertinos e noturnos, em comparação com o modelo anterior, marcado por cursos matutinos. Esta mudança possibilitou a inclusão de um número maior de estudantes-trabalhadores. Estes estudantes enfrentam a jornada de trabalho em paralelo à jornada de estudos. Em muitos casos, alguns domínios podem estar prejudicados, como é o caso do domínio biológico, influenciado por um número insuficiente de horas de sono e repouso, por exemplo.

Considerando o contexto apresentado, esta pesquisa teve como objetivos identificar o nível de qualidade de vida entre estudantes de enfermagem de um curso de graduação em enfermagem de uma universidade privada da Zona Sul da Cidade de São Paulo, estabelecendo como variável de comparação o período de estudos.

\section{Métodos}

Tratou-se de um estudo correlacional no qual as variáveis sobre a QV foram comparadas. O estudo correlacional é usado para examinar a relação entre duas ou mais variáveis (LOBIONDOWOOD; HABER, 2001). O estudo foi realizado no campus universitário de uma universidade privada da Zona Sul da Cidade de São Paulo, tendo aprovação pelo Comitê de Ética em Pesquisa da Universidade de Santo Amaro mediante o parecer número 134/2009. Fizeram parte da pesquisa estudantes do Curso de Enfermagem regularmente matriculados na instituição de ensino. A amostra foi composta pela técnica não-probabilística de conveniência, quando os estudantes foram abordados com a apresentação da Carta de Informação. Após a anuência, os participantes assinaram o Termo de Consentimento Livre e Esclarecido.

Foi utilizado o instrumento WHOQOL-bref (THE WHOQOL GROUP, 1995) por ser considerado um instrumento validado e que abrange o conteúdo o qual foi objeto desta pesquisa. A ausência de um instrumento que avaliasse qualidade de vida, com uma perspectiva internacional, fez com que a Organização Mundia de Saúde (OMS) construísse um Grupo de Qualidade de Vida 
com a finalidade de desenvolver instrumentos capazes de fazê-lo dentro de uma perspectiva transcultural (FLECK, 2000).

O WHOQOL-bref é composto por 26 questões que dentro de cinco domínios quais sejam: físico, psicológico, nível de independência, relações sociais e ambiente. Este questionário utiliza o sistema com níveis de resposta, como exemplo: nada $=1$; muito pouco $=2$; médio $=3$; muito $=4$ e, completamente $=5$, produzindo um escore. A coleta de dados ocorreu durante o período das aulas, em horários combinados com a coordenação do curso e de acordo com a autorização dos professores nas salas de aula.

Para a análise dos dados foram formados os dois grupos segundo o período de estudos: matutino e noturno. Para a análise dos dados referentes aos domínios do WHOQOL-bref foi desenvolvida uma planilha no aplicativo MS Excel® para o cálculo dos escores, seguindo o modelo de Pedroso, Pilatti e Reis (2009). Para classificar os resultados foram considerados os seguintes níveis: < 25\%, QV ruim; de 25 a 75\%, QV intermediária ou boa e > 75\%, QV ótima (Quadro 1).

Após a obtenção dos dados descritivos, foi realizada a estatística não-paramétrica. Foram produzidas as médias dos escores por cada domínio, segundo o período e feita comparação por meio do teste do Qui-quadrado (SIEGEL; CASTELLAN JÚNIOR, 2006).

Quadro 1: Critérios utilizados no instrumento para o estabelecimento das categorias

\begin{tabular}{|l|c|c|c|}
\hline Escala & $\mathbf{2 5 \%}$ & $\mathbf{2 5} \mathbf{- 7 5 \%}$ & $\mathbf{7 5 \%}$ \\
\hline Intensidade & Nada & mais ou menos & Bastante \\
\hline Avaliação & Insatisfeito & $\begin{array}{c}\text { nem satisfeito, } \\
\text { nem insatisfeito }\end{array}$ & Satisfeito \\
\hline Capacidade & Ruim & mais ou menos & muito bem \\
\hline Frequência & Nenhuma & apenas algumas & o suficiente \\
\hline
\end{tabular}

Fonte: Pesquisa de campo (2010)

\section{Resultados}

\subsection{Perfil da Amostra}

A pesquisa foi realizada nos meses de fevereiro a março de 2010 , tendo a participação de 230 alunos (105 do período matutino e 125 do período noturno). Quanto ao sexo, 18,3\% eram do sexo masculino e $81,7 \%$ do sexo feminino. Maiores detalhes em relação ao perfil dos participantes estão apresentados na Tabela 1.

No Brasil vem crescendo o interesse pelo tema da QV (SEIDI; ZANON, 2004). A QV deve ser valorizada no processo de formação dos acadêmicos de enfermagem e é necessário criar mecanismos de suporte que instrumentalizem os acadêmicos para o enfrentamento das inúmeras situações penosas que vivenciam no processo de sua formação (TAVARES et al., 1995).

Neste estudo, embora sendo considerada a abordagem para uma população jovem, o estudo sobre QV assume um importante papel como a detecção de fatores de risco para doenças, como destacado no estudo de Alves e Marques (2009). No presente estudo, a faixa etária de maior frequência foi à de 21 a 25 anos $(41,3 \%)$, com predomínio de estudantes no período noturno conforme apresentado na Tabela 1. Embora o conceito geral de QV envolva a questão da saúde, sabemos que a QV difere de pessoa para pessoa e envolve outros aspectos também importantes como: satisfação e disposição para o trabalho (PASCOAL; DONATO, 2005).

Tabela 1 - Perfil dos participantes da pesquisa $(n=230)$

\begin{tabular}{|c|c|c|c|c|c|c|}
\hline \multirow{2}{*}{ Variável } & \multicolumn{2}{|c|}{ Matutino } & \multicolumn{2}{|c|}{ Noturno } & \multicolumn{2}{|c|}{ Total } \\
\hline & $f i$ & $\%$ & $f i$ & $\%$ & $f i$ & $\%$ \\
\hline \multicolumn{7}{|l|}{ Idade } \\
\hline$<20$ & 18 & 17,1 & 22 & 17,6 & 40 & 17,4 \\
\hline $21-25$ & 42 & 40,0 & 53 & 42,4 & 95 & 41,3 \\
\hline $26-30$ & 28 & 26,6 & 26 & 20,8 & 54 & 23,5 \\
\hline $31-35$ & 9 & 8,5 & 10 & 8,0 & 19 & 8,3 \\
\hline
\end{tabular}




\begin{tabular}{|c|c|c|c|c|c|c|}
\hline $36-40$ & 5 & 4,7 & 10 & 8,0 & 15 & 6,5 \\
\hline$>40$ & 3 & 2,8 & 4 & 3,2 & 7 & 3,0 \\
\hline \multicolumn{7}{|l|}{ Sexo } \\
\hline Masculino & 13 & 12,3 & 29 & 23,2 & 42 & 18,3 \\
\hline Feminino & 92 & 87,6 & 96 & 76,8 & 188 & 81,7 \\
\hline \multicolumn{7}{|l|}{ Período (Semestre) } \\
\hline $2^{\circ}$ & - & 0,0 & 15 & 12,0 & 15 & 6,5 \\
\hline $3^{\circ}$ & 8 & 7,6 & 18 & 14,4 & 26 & 11,3 \\
\hline $5^{\circ}$ & 32 & 30,5 & 25 & 20,0 & 57 & 24,8 \\
\hline $6^{\circ}$ & 0 & 0,0 & 24 & 19,2 & 24 & 10,4 \\
\hline $7^{\circ}$ & 65 & 61,9 & 9 & 7,2 & 74 & 32,2 \\
\hline $8^{\circ}$ & - & 0,0 & 34 & 27,2 & 34 & 14,8 \\
\hline \multicolumn{7}{|c|}{ Trabalha (paralelo ao estudo) } \\
\hline Sim & 69 & 65,7 & 85 & 68,0 & 154 & 67,0 \\
\hline Não & 36 & 34,2 & 40 & 32,0 & 76 & 33,0 \\
\hline
\end{tabular}

Fonte: Pesquisa de campo (2010)

Soma-se a isto a questão do aluno trabalhador, como verificado, 67,0\% trabalha paralelamente à atividade de estudo. $\mathrm{O}$ trabalho associado aos estudos é um fator que pode impactar a QV. Por outro lado, conclui-se que a análise quantitativa dos dados referentes à pesquisa realizada, um estudo demonstrou que não existe relação entre trabalho e período de estudo (CARELLI; SANTOS, 1998). Ainda em relação ao perfil, verificou-se que 54,3\% dos alunos optou por estudar no período noturno. Com relação à esta variável, Gambardella e Gotlieb (1998), justificam que o jovem estudante tem um gasto energético maior quando seus estudos ocorrem no período noturno e isto pode representar uma influência na QV.

A falta de atividade física ou o sedentarismo entre os universitários pode ocorrer em detrimento da sobrecarga da carga horária em sala de aula e necessidade de despender seu tempo livre com atividades também relacionadas à universidade ou aos estudos (SOARES; CAMPOS, 2008). A atividade física regular oferece contribuição à QV (PASCOAL; DONATO, 2005), por proporcionar um estilo de vida saudável. Sendo assim, um tópico ou domínio com baixo desempenho passa a interferir no outro, como por exemplo: sono/repouso (SOARES; CAMPOS, 2008).

\subsection{Análise da Qualidade de Vida do Estudante}

Conforme apresentado na Tabela 2, quanto ao Domínio Físico, a categoria QV intermediária apresentou índices maiores para os alunos do período matutino em comparação com os alunos do período noturno. Na categoria QV ótima, alunos do período matutino apresentaram resultados inferiores aos do período noturno. No teste do Qui-quadrado, não houve diferença estatisticamente significante (QV $p=0,3323$ ). Nessa perspectiva, é importante que sejam criados espaços para que os estudantes possam reativar sua energia (JORGE, 1996).

Tabela 2 - QV dos estudantes de enfermagem e os domínios do WHOQOL-bref.

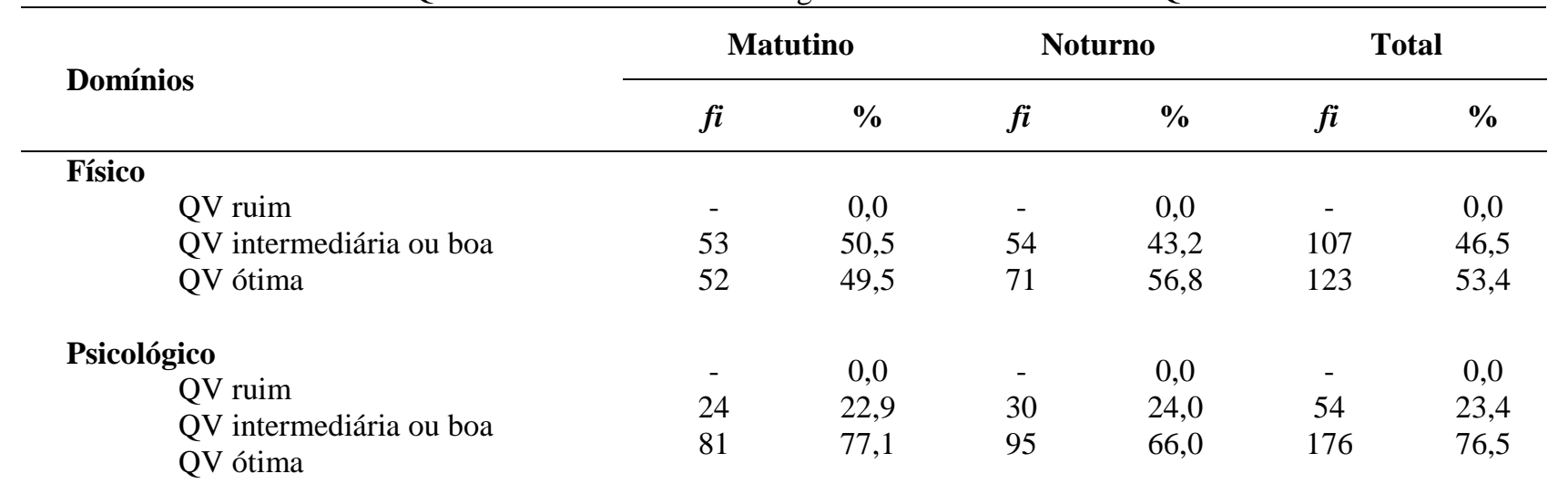




\section{Nível de independência}

$\mathrm{QV}$ ruim

QV intermediária ou boa

QV ótima

Relações sociais

QV ruim

QV intermediária ou boa

QV ótima

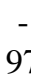

97

8

$-$

65

Ambiente

QV ruim

QV intermediária ou boa

QV ótima

$\begin{array}{cccccc}- & 0,0 & - & 0,0 & - & 0,0 \\ 87 & 92,4 & 118 & 94,4 & 215 & 93,4 \\ 8 & 7,6 & 7 & 5,6 & 15 & 6,5 \\ & & & & & \\ - & 0,0 & - & 0,0 & - & 0,0 \\ 0 & 38,1 & 44 & 35,2 & 84 & 36,5 \\ 65 & 61,9 & 81 & 64,8 & 146 & 63,4 \\ & & & & & \\ & & & & & \\ - & 0,0 & - & 0,0 & - & 0,0 \\ 2 & 59,0 & 64 & 51,2 & 126 & 54,7 \\ 3 & 41,0 & 61 & 48,8 & 104 & 45,2\end{array}$

0,0

3,4

6,5

0,0

36,5

63,4

0,0

45,2

Fonte: Pesquisa de campo (2010)

Em relação ao Domínio Psicológico, a categoria QV intermediária teve resultados semelhantes para os estudantes dos dois períodos (noturno $24,0 \%$ e matutino $22,9 \%$ ). Na categoria QV ótima os estudantes do período matutino tiveram resultados maiores (matutino $77,1 \%$ e noturno $66,0 \%)$. Vale destacar que os estudantes do período matutino relataram maior frequência de sentimentos negativos em relação aos estudantes do período noturno. A análise comparativa resultou em um valor estatisticamente significante $(p=0,0035)$ no teste do Qui-quadrado.

Estes resultados do período matutino foram abordados segundo o semestre de estudos. Verificou-se que os alunos do $7^{\circ}$. Semestre do período matutino tiveram um percentual maior de sentimentos negativos na seguinte frequência: nunca 3,8\%, às vezes $45,7 \%$ e sempre $12,3 \%$. Embora estes resultados não tenham sido semelhantes para a turma do $7^{\circ}$. Semestre do período noturno e, buscando entender este resultado e sua influência na QV, atribuiu-se como causa o fato de que neste semestre inicia-se a fase final da graduação. Concernente a isso, embora os contextos dos cursos de graduação em enfermagem sejam permeados por situações conflitantes que podem gerar emoções e sentimentos desagradáveis os índices de QV dos estudantes apresentam-se altos (KAWAKAME, MIYADAHIRA, 2005).

Os estudantes, em razão da adaptação ao novo modelo de vida imposto pelo mundo universitário, estariam mais susceptíveis a perturbações emocionais (CERCHIARI; CAETANO; FACCENDA, 2005). Outros fatores podem também contribuir para aumentar os sentimentos negativos dos alunos, dentre eles a preocupação com o mercado de trabalho (JORGE, 1996). Vale ressaltar que na medida em que o acadêmico permanece na universidade há um aumento da tensão ou estresse psíquico, distúrbios psicossomáticos, falta de confiança na capacidade de desempenho e auto-eficácia, indicando assim uma diminuição da saúde mental geral (JORGE, 1996), incluindo a possibilidade de desenvolver a Síndrome de Burnout (TOMAZELA; GROLLA, 2007). Quanto ao domínio Nível de Independência, a categoria QV intermediária dos estudantes do período noturno foi de $94,4 \%$ e dos estudantes do período matutino 92,4\%. Considerando QV ótima, os estudantes do período matutino aparecem com 7,6\% contra 5,6\% dos estudantes do período noturno. Na análise estatística com o teste do Qui-quadrado o resultado foi não-significante $(p=0,729)$.

Referente ao Domínio Relações Sociais, na categoria QV intermediária, os estudantes do período matutino apresentaram $38,1 \%$, e os estudantes do período noturno $35,2 \%$. Avaliando a categoria QV ótima os estudantes do período noturno tiveram $64,8 \%$ e os estudantes do período matutino $61,9 \%$. Na análise estatística o valor foi não-significante $(p=0,7518)$.

$\mathrm{Na}$ avaliação do Domínio Ambiente, a categoria QV intermediária dos estudantes do período matutino foi de $59,0 \%$ e a dos estudantes do período noturno de $51,2 \%$. Em relação à categoria QV ótima, os estudantes do período noturno obtiveram $48,8 \%$ e os estudantes do período matutino $41,0 \%$. Na análise estatística Ao teste de Qui-quadrado não houve significância pelo valor obtido $(p=0,2899)$. Nota-se, que em relação aos cinco domínios abordados na pesquisa, nenhum período obteve resultado de má QV, percebe-se o quanto são semelhantes os valores obtidos de cada 
período por domínio em relação à frequência de respostas.

A OMS aponta a vivência de pessoas em situações de estresse como situação prioritária para a mensuração da QV (FLECK, 2000), pois a possibilidade de se desenvolver a Síndrome de Burnout e estresse a partir de um ambiente que exerça pressão nos indivíduos é muito grande (TOMAZELA, GROLLA, 2007). A análise global que responde a questão principal deste estudo está apresentada no Gráfico 1.

Figura 1 - Comparação das médias das categorias de QV, segundo período de estudo

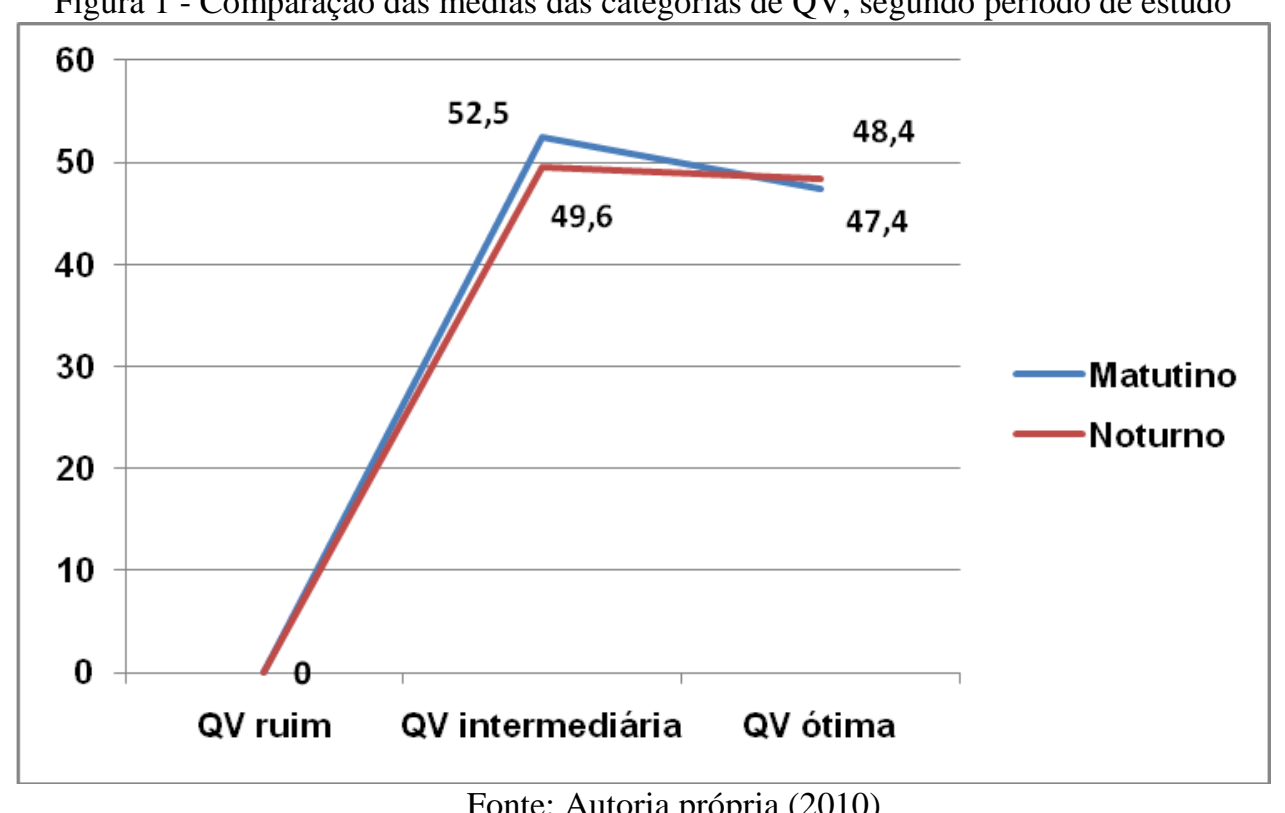

Fonte: Autoria própria (2010)

Ao analisar os resultados de todos os domínios somados por escala do período matutino e noturno, verificou-se que na escala $\mathrm{QV}$ intermediária os estudantes do período matutino tiveram resultados superiores ao do período noturno $(52,5 \%$ a $49,6 \%)$ e na escala QV ótima, os estudantes do período noturno é que apresentaram resultados superiores $(48,4 \%$ a $47,4 \%)$.

\section{Conclusões}

Frente aos resultados apresentados, pode-se concluir que os estudantes participantes da pesquisa têm QV intermediária ou boa (matutino=52,5, noturno $=49,6$ ) ou $\mathrm{QV}$ ótima (matutino $=47,4$, noturno $=48,4)$. As variações decorreram do domínio avaliado, sem haver discrepâncias na comparação quanto ao período de estudos, não havendo diferença estatisticamente significante na QV.

Sendo a QV um construto interdisciplinar essencial a todos, percebe-se o quanto é indispensável na formação do graduando, futuro enfermeiro; pois como todo profissional da área da saúde, esse indicador refletirá na assistência de enfermagem ao paciente e na qualidade de vida de seus sujeitos. Novos estudos são necessários com a utilização de outros instrumentos que permitam captar diferenças não sensíveis ao WHOQOL-bref.

\section{Referências}

ALVES, A.; MARQUES, I. R. Fatores relacionados ao risco de doença arterial coronariana entre estudantes de enfermagem. Revista Brasileira de Enfermagem, Brasília, v. 62, n. 6, p. 883-888, mar./abr., 2009.

ÂNGELO, M. Vivendo uma prova de fogo: as experiências iniciais de uma aluna de enfermagem. 1989. 133 f. Dissertação (Mestrado em Psicologia). Instituto de Psicologia, Universidade de São Paulo, São Paulo. 
CAPLAN, G. Princípios de psiquiatria preventiva. Rio de Janeiro: Editora Zahar, 1980.

CARELli, M. J. G.; SANTOS, A. A. A. Condições temporais e pessoais de estudo em universitários. Psicologia Escolar e Educacional, São Paulo, v. 2, n. 3, p. 265-278, mai./ago., 1998.

CHERCHIARI, E. A. N.; CAETANO, D.; FACCENDA, O. Prevalência de transtornos mentais menores em estudantes universitários. Estudos de Psicologia, Natal, v. 10, n. 3, p. 412-420, set./dez., 2005.

CIAMPONE, M. H. T. Grupo operativo: construindo bases para o ensino e a prática na enfermagem. 1998. 156 f. Tese (Livre Docência em Enfermagem) - Escola de Enfermagem, Universidade de São Paulo, São Paulo.

FIGUEIREDO, R. M.; OLIVEIRA, M. A. P. Necessidades de estudantes universitários para implantação de um serviço de orientação em saúde mental. Revista Latino-Americana de Enfermagem, Ribeirão Preto, v. 3, n. 1, p. 5-18, jan./jun., 1995.

FLECK, M. P. A. O instrumento de avaliação de qualidade de vida da Organização Mundial da Saúde (WHOQoL-100): características e perspectivas. Ciência \& Saúde Coletiva, Rio de Janeiro, v. 5, n. 1, p. 33-8, jan./jun., 2000.

GAMBARDELLA, A. M. D.; GOTLIEB, S. L. D. Dispêndio energético de adolescentes estudantes do período noturno. Revista de Saúde Pública, São Paulo, v. 32, n. 5, p. 413-419, out./nov., 1998.

JOLY, M. C. R. A.; SANTOS, A. A. A.; SISTO, F. F. Questões do cotidiano universitário. São Paulo: Casa do Psicólogo; 2005.

JORGE, M. S. B. Situações vivenciadas pelos alunos de enfermagem durante o curso no contexto universitário: apontadas como norteadoras de crises. Revista da Escola de Enfermagem da USP, São Paulo, v. 30, n. 1, p. 138-148, jan./abr., 1996.

KAWAKAME, P. M. G.; MIYADAHIRA, A. M. K. Qualidade de vida de estudantes de graduação em enfermagem. Revista da Escola de Enfermagem da USP, São Paulo, v. 39, n. 2, p. 164-172, abr./jun., 2005.

LOBIONDO-WOOD, G.; HABER, J. Pesquisa em enfermagem - métodos, avaliação crítica e utilização. Rio de Janeiro: Guanabara-Koogan, 2001.

NETTO, A. R. Qualidade de vida: compromisso histórico da epidemiologia. In: LIMA E COSTA, M. F. L.; SOUSA, R. P. (Org.). Qualidade de vida: compromisso histórico da epidemiologia. Belo Horizonte: Coppmed/Abrasco, 1994. p. 11-8.

PASCOAL, M.; DONATO, J. C. Aspectos psicofísicos e sócio-culturais da qualidade de vida. Movimento \& Percepção, Espírito Santo do Pinhal, v. 5, n. 6, p. 165-168, nov./dez., 2005.

PEDROSO, B.; PILATTI, L. A.; REIS, D. R. Cálculo dos escores e estatística descritiva do WHOQOL-100 utilizando o Microsoft Excel. Revista Brasileira de Qualidade Vida, Ponta Grossa, v. 1, n. 1, p. 23-32, jan./jun., 2009. 
SEIDI, E. M. F.; ZANON, C. M. L. C. Qualidade de vida e saúde: aspectos conceituais e metodológicos. Cadernos de Saúde Pública, Rio de Janeiro, v. 20, n. 2, p. 580-508, mar./abr., 2004.

SIEGEL, S. E.; CASTELLAN JÚNIOR, N. J. Estatística não-paramétrica para ciências do comportamento. 2. ed. Porto Alegre: Artmed, 2006.

SOARES, R. D. O. P.; CAMPOS, L. F. Estilo de vida dos estudantes de enfermagem de uma universidade do interior de Minas Gerais. Cogitare Enfermagem, Curitiba, v. 13, n. 2, p. 227-234, abr./jun., 2008.

TAVARES, M. S. G. et al. O perfil do aluno ingressante nos cursos superiores de enfermagem do Estado de São Paulo - 1993. Revista Paulista de Enfermagem, São Paulo, v. 14, n. 2/3, p. 55-65, abr./dez., 1995.

TOMAZELA, N.; GROLLA, P. P. Síndrome de burnout. In: SIMPÓSIO DE ENSINO DE GRADUAÇÃO, 5., 2007, Piracicaba, Brasil. Anais... Piracicaba: UNIMEP, 2007.

THE WHOQOL GROUP. The World Health Organization Quality of Life Assessment (QOL): position paper from the World Health Organization. Social Science and Medicine, Leicester, v. 41, n. 10, p. 1403-1409, nov., 1995. 